\title{
Comparación entre mediciones de velocidad obtenidas con los equipos GPS y Pistola Láser
}

Comparison of speed measurement data obtained with GPS and laser gun devices

Fecha de entrega: 7 de agosto 2012 Fecha de aceptación: 6 de septiembre 2013

\section{Tomás Echaveguren, Álvaro Díaz y Daniela Arellano}

Departamento de Ingeniería Civil, Universidad de Concepción, Edmundo Larenas 219-Interior, Casilla 160-C, Correo 3, CP 4070409,Concepción, Chile, techaveg@udec.cl, adiaz@udec.cl, danarellano@udec.cl

La modelación de relaciones entre la velocidad de operación y la geometría de las carreteras requiere contar con registros de velocidad precisos y exactos. Existen dispositivos pasivos y activos para la medición de velocidad, entre los cuales resaltan el GPS y la pistola láser, dado su bajo costo y fácil implementación en terreno. Los estudios de precisión de estos equipos requieren además un control preciso de la velocidad de referencia del vehículo de prueba, lo cual se logra con el tacómetro. Por otro lado, el uso combinado de GPS y pistola láser, requiere conocer la relación de lecturas de velocidad entre ambos. En este trabajo se estudió en terreno la relación entre las lecturas de velocidad obtenidas con tacómetro, GPS y pistola láser. Para ello se implementó en terreno un experimento que consideró mediciones con ambos equipos a velocidades entre 20 y $120 \mathrm{~km} / \mathrm{h}$. Los datos se procesaron y depuraron a fin de eliminar datos anómalos. Los datos de GPS fueron procesados mediante el filtro de Kalman para acoplar lecturas de velocidad y posición. Luego del análisis estadístico, se elaboraron correlaciones entre lecturas de velocidad obtenidas con tacómetro y GPS y con pistola láser y GPS. Se concluye que las diferencias entre las lecturas de velocidad del tacómetro $y$ del GPS son estadisticamente diferentes, variando entre 3 y $10 \mathrm{~km} / \mathrm{h}$, y que las lecturas entre pistola láser y GPS son estadísticamente iguales con una diferencia sistemática de $0.5 \mathrm{~km} / \mathrm{h}$.

Palabras clave: velocidad, GPS, pistola láser
The modelling of relationships between operating speed and highway geometry needs accurate and exact speed records. There exist passive and active devices for speed measuring. Among those devices, the GPS and laser guns highlight considering its low cost and its in-field easy-touse speed measurement. Accuracy studies of those devices need a good control of the probe vehicle reference speed, which is achieved by using the tachometer. On the other side, combined use of the GPS and the laser gun need to know the relationship between speed records of both devices. This paper presents an in-field study of the relationships between speed records obtained with tachometer, GPS and laser gun. The experiment was carried out on a road test section. The speed measurements performed with those devices ranged between 20 and $120 \mathrm{~km} / \mathrm{h}$. Speed data were processed and debugged to erase outliers. The GPS speed data were processed using Kalman filter to assemble speed data and position data. After statistical analysis, correlations between tachometer and GPS and between laser gun and GPS were obtained. It is concluded that the differences of speed records between tachometer and GPS are statistically significant, ranging between 3 and $10 \mathrm{~km} / \mathrm{h}$. Whereas the speed records obtained with laser gun and GPS were statistically equal and it was identified a systematic difference between both devices of $0.5 \mathrm{~km} / \mathrm{h}$.

Keywords: speed, GPS, laser gun

ingeniería de carreteras: para estudios de velocidades límite, para estudios de mejoramiento de trazado, análisis de consistencia y para modelación de relaciones causales
Los datos de velocidad de operación obtenidos en terreno son necesarios para realizar diversos estudios en 
entre velocidad de operación y geometría del trazado de las carreteras. Existen diversas tecnologías de medición de velocidad en carreteras. Dependiendo del uso que se le dé a los registros de velocidad, algunas son más adecuadas que otras en términos de resolución, precisión, exactitud, cantidad de datos, cobertura geográfica y costos de los sistemas de medición (AASHTO, 2009). En Chile, las mediciones de velocidad en carreteras no son de carácter rutinario, sino que proyecto a proyecto, con excepción de las mediciones continuas que se realizan en rutas concesionadas. Los métodos usados para estos efectos son básicamente conteos manuales de velocidad media espacial o velocidad media temporal, mediciones con sistemas láser, como el usado en este estudio y sensores de piso fijos, que se encuentran embebidos en el pavimento y que son usados esencialmente en vías concesionadas.

Para modelación del efecto de la geometría sobre la velocidad, los métodos de medición más usados se pueden clasificar en intrusivos y no intrusivos desde el punto de vista de la percepción del conductor. Los métodos intrusivos son aquellos en los cuales el conductor percibe que está siendo observado y por tanto modifica su conducta. Dentro de los métodos intrusivos se encuentra la pistola láser, que permite obtener datos puntuales en sectores específicos del trazado. Los métodos no intrusivos son aquellos que permiten obtener datos de velocidad sin que el conductor perciba que está siendo medido, por lo cual no modifica sustancialmente su conducta. Dentro de esta categoría se encuentran los dispositivos GPS, que permiten obtener datos de velocidad en largas distancias.

En Chile, Echaveguren y Sáez (2001), Echaveguren y Basualto (2003) y Vargas (2008) han usado la pistola láser para calibrar modelos de velocidad. Asimismo, Al-Masaied et al. (1995) y Abdelwahab et al. (1998) han calibrado modelos de velocidad en curvas horizontales usando pistola láser para registrar velocidades de operación. El logger GPS por su parte, ha sido usado preferentemente para estudiar perfiles de velocidad de camiones, calibrar modelos que relacionan velocidad con geometría y análisis de consistencia (Memon et al., 2008; Echaveguren, 2012; Echaveguren et al., 2012a) y para estudios de tiempo de viaje y nivel de servicio, tanto en carreteras como en vías urbanas (Li, 2009; Schuessler y Axhausen, 2009; Pérez et al., 2010; Bhuyan y Rao, 2012).
Los estudios de precisión y exactitud de un sistema de medición de velocidad basado en equipos como la pistola láser y loggers GPS, utilizan vehículos de prueba que circulan a diferentes velocidades de referencia. Habitualmente la velocidad de referencia se le informa al conductor del vehículo de prueba, quien la controla con ayuda del tacómetro. Estudios de campo realizados por Echaveguren et al. (2011 y 2012b) han mostrado que existen diferencias entre las velocidades reales de circulación y aquella registrada con el tacómetro. Esto induce un sesgo que tiende a magnificar la variabilidad, subestimando así el valor de la precisión calculada para los equipos de medición.

Por otro lado, cuando se realizan mediciones de velocidad con el logger GPS aplicando el método de seguimiento vehicular, existe un error de estimación debido a la variabilidad del headway entre el vehículo líder y el seguidor, el cual sólo puede estimarse con sistemas complementarios de medición de velocidad, siendo la pistola láser uno de los equipos útiles para esos efectos. Sin embargo, es necesario previamente contar con una homologación de medidas de velocidad entre ambos equipos, a partir de la cual establecer una base de comparación que permita medir el margen de error en la estimación de velocidad al usar el seguimiento vehicular.

\section{Objetivos y alcances}

El objetivo de este trabajo es contrastar tres métodos de medición de velocidad en terreno: tacómetro digital, pistola láser y logger GPS, con el fin de establecer el margen de error relativo esperable de las lecturas de velocidad entre esos equipos. Conocer el margen de error del tacómetro digital del vehículo de medición permite establecer con mayor certeza la verdadera velocidad de referencia cuando se desea realizar medidas repetidas a velocidades controladas, como por ejemplo para calibrar modelos de cálculo de límites de velocidad en curvas (véase Echaveguren y Vargas-Tejeda, 2013). Por otro lado, permite identificar cual es la exactitud relativa de un equipo en contraste con un equipo de referencia para determinar con mayor claridad el margen de error de mediciones que se realicen mediante seguimiento vehicular.

El trabajo consideró en primera instancia una revisión 
del estado del arte en cuanto a mediciones de velocidad con pistola láser y GPS, presentado en la introducción. Posteriormente se presenta el diseño experimental, el cual incluye el cálculo del tamaño muestral, una descripción de los equipos utilizados, la selección del tramo de prueba y el procedimiento de medición en terreno. Posteriormente se discute el procesamiento de datos, que incluye la eliminación de datos anómalos, el filtrado de la señal de velocidad registrada por el GPS y el acople de mediciones de velocidad realizadas con el GPS y la pistola láser, alrededor de la baliza de medición. Posteriormente, en el análisis de resultados se discute la relación entre las velocidades registradas por el tacómetro del vehículo y las lecturas de velocidad del GPS en las balizas de medición, para luego desarrollar el análisis estadístico de las lecturas de velocidad obtenidas con el dispositivo GPS y la pistola láser. Finalmente se presentan las conclusiones y recomendaciones emanadas del trabajo.

\section{Diseño experimental}

\section{Descripción de los equipos de medición de velocidad}

Los equipos utilizados en este trabajo permiten estimar velocidad instantánea con distintas tecnologías y métodos. Por un lado, el dispositivo GPS permite obtener lecturas de velocidad, aceleración, posición, azimut, radio de curvatura cada 0.1 s. Dependiendo de la velocidad de medición, el equipo puede obtener ente 1800 y 500 datos/km para un rango de velocidades entre 20 y $120 \mathrm{~km} / \mathrm{h}$. Puesto que el equipo es portátil y se monta en un vehículo de prueba, las mediciones de velocidad se realizan usando una muestra de conductores o bien aplicando la técnica de seguimiento vehicular.

El dispositivo láser por su parte, es un equipo que permite medir velocidad instantánea de un vehículo desde un punto fijo o desde un vehículo en movimiento. Posee la limitante que los registros de velocidad no están georeferenciados, por lo cual se requiere establecer una baliza de referencia para identificar la sección del camino donde se activa la lectura. La Tabla 1 muestra una comparación entre el equipo GPS y láser en términos de la medición de velocidad.

Si bien con el equipo láser se pueden obtener lecturas en secciones largas de caminos, sus limitaciones propias y las de la forma de registro de velocidad, impiden obtener una densidad de datos/km adecuada. En este sentido el dispositivo GPS posee la capacidad de almacenamiento automático, en tanto que la pistola láser requiere personal de apoyo para registrar las velocidades.

Tabla 1: Características de los equipos GPS y láser (Kustom Signals, 2002; Racelogic, 2008)

\begin{tabular}{|l|l|l|}
\hline Característica & Logger GPS & Pistola Láser \\
\hline Tipo de medición & Continua & Puntual \\
\hline Rango de medición, $\mathrm{km} / \mathrm{h}$ & $0.01-1600$ & $10-320$ \\
\hline Precisión, $\mathrm{km} / \mathrm{h}$ & 0.2 & 2 \\
\hline Resolución de medición, $\mathrm{km} / \mathrm{h}$ & 0.01 & 1 \\
\hline Tiempo de captura de dato, $\mathrm{s}$ & 0.1 & 0.3 \\
\hline Geo-referencia datos & $\mathrm{si}$ & $\mathrm{no}$ \\
\hline
\end{tabular}

\section{Selección de tramo de medición}

Con el fin de replicar las condiciones reales de operación de los equipos GPS y pistola láser, se optó por identificar tramos de medición en caminos rurales bidireccionales. Para ello, se predefinieron criterios de selección con el fin de controlar los errores que no se deben a los equipos y que son propios de las características físicas y operativas del tramo de medición. De este modo, los criterios empleados para seleccionar el tramo de medición fueron:

- Pendiente longitudinal menor al $2 \%$ para eliminar efecto de la pendiente longitudinal en la velocidad,

- flujo vehicular menor a 2500 vehículos/día-año por motivos de seguridad,

- pavimento en buen estado con demarcación lateral en buen estado,

- alineamiento horizontal recto,

- berma y despeje lateral apropiado,

- Sin señal vertical de velocidad límite,

- espacio disponible para realizar giros y detenciones sin afectar la circulación en la vía, y

- longitud adecuada para alcanzar las distintas velocidades requeridas por el ensayo.

Utilizando estos criterios, se identificó en terreno un tramo de la ruta que une Cabrero y Yungay en la región del Bío Bío, comprendido entre los km 5.0 y 8.0 el cual se ilustra 
en la Figura 1. En el tramo seleccionado se escogió un segmento de $1.4 \mathrm{~km}$ que contaba con visibilidad suficiente para realizar el experimento. Posteriormente se construyó en terreno la configuración de segmento de medición en base a mediciones de prueba.

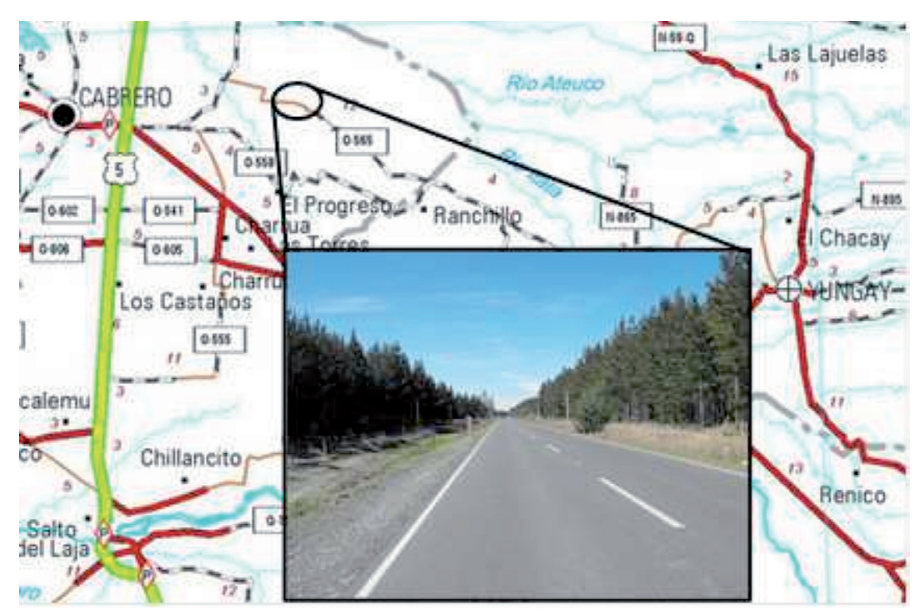

Figura 1: Localización de tramo seleccionado para realizar el experimento

\section{Cálculo de tamaño de la muestra}

Este cálculo se realizó para determinar el número mínimo de pasadas del vehículo de prueba a la velocidad de referencia y el número mínimo de puntos de control necesarios para realizar la medición, para asegurar el control de los errores tipo I y II. Para ello se utilizó el análisis de potencia estadística de Cohen (1988), utilizando para ello el software GPower V3.1. Considerando que las correlaciones poseen solo 1 predictor, se estimó que para un efecto tamaño grande $\left(\mathrm{f}^{2}=0.35\right)$ una confiabilidad del $5 \%$, se requiere una tamaño muestral $\mathrm{N}=33$ para alcanzar una potencia estadística del $90 \%$ y N $=25$ para alcanzar una potencia estadística del $80 \%$. Desde el punto de vista del experimento, esto se traduce en lo siguiente:

a) Para correlacionar la velocidad del tacómetro con la velocidad registrada con el GPS, con 3 puntos de control, se requieren como mínimo 8 pasadas a las velocidades de referencia desde los $20 \mathrm{~km} / \mathrm{h}$.

b) Para correlacionar la velocidad registrada con el GPS y la pistola láser, con 3 puntos de control se requieren por cada uno 6 lecturas con pistola láser. Esto asegura que en cada pasada por el tramo se cuenta con al menos 18 puntos de medición para las velocidades de referencia de
$20,40,60,80,100$ y $120 \mathrm{~km} / \mathrm{h}$. De este modo, para el experimento desarrollado en el tramo de prueba se cuenta con un tamaño de muestra mínimo adecuado para realizar las correlaciones entre sistemas de medición de velocidad.

\section{Mediciones preliminares}

Se realizaron mediciones de prueba considerando los 3 puntos de control espaciados cada $50 \mathrm{~m}$, con el fin de verificar si dicha distancia resultaba adecuada o no, de acuerdo a las velocidades de referencia a usar en las mediciones. Se determinó que una separación entre puntos de control de $50 \mathrm{~m}$ era insuficiente para que el operador de la pistola láser pudiese registrar mediciones sucesivas sobre todo a velocidades elevadas. Después de sucesivas mediciones se determinó que la distancia adecuada entre puntos de control era entre 70 y $100 \mathrm{~m}$. De este modo, se utilizaron balizas espaciadas cada $100 \mathrm{~m}$ emplazadas al costado de la berma derecha para representar cada punto de control. Luego se realizaron mediciones de prueba para estimar la longitud necesaria para acelerar y decelerar, de tal manera que el vehículo de prueba pase por los puntos de control a una velocidad constante y controlar así la variabilidad temporal y espacial de la velocidad. También se verificó la distancia máxima a la cual el operador de la pistola alcanza a distinguir cuando el vehículo atraviesa la baliza seleccionada. Con estos ensayos se configuró finalmente el tramo de medición para realizar el experimento.

\section{Configuración de tramo de medición en terreno}

La configuración en terreno del experimento consideró un tramo de aceleración de $400 \mathrm{~m}$, un tramo de estabilización de velocidad de $300 \mathrm{~m}$, tres puntos de control espaciados $100 \mathrm{~m}$ cada uno, el operador ubicado a $70 \mathrm{~m}$ del último punto de control y un tramo de deceleración de $300 \mathrm{~m}$. Esto arroja una longitud total de $1270 \mathrm{~m}$. La Figura 2 muestra la localización del operador de la pistola láser respecto de los puntos de control de velocidad. Dependiendo de la distancia entre el operador y la baliza, el ángulo de medición de la pistola láser varió entre $2^{\circ}$ y $8^{\circ}$ respecto del eje de la pista de circulación del vehículo de ensayo, dependiendo del punto de control sobre el cual se mide la velocidad. La Figura 3 muestra la configuración general del experimento realizado en terreno. 


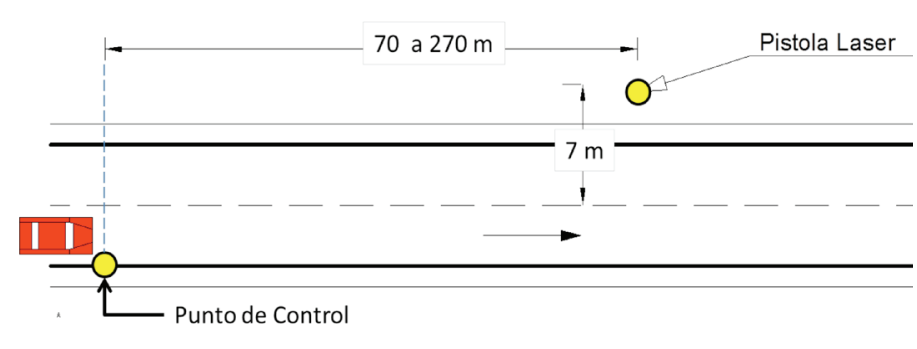

Figura 2: Ubicación de operador de pistola láser respecto de puntos de control.

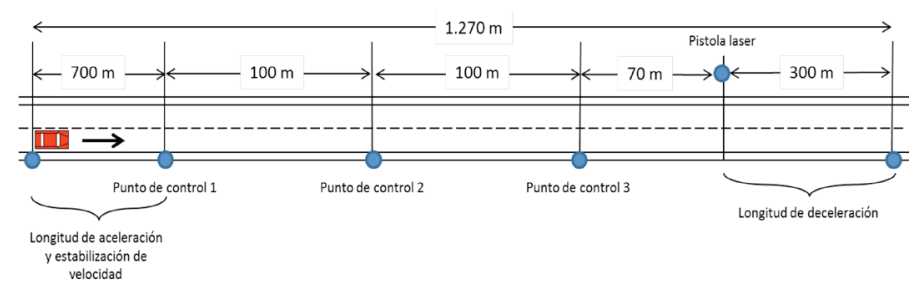

Figura 3: Esquema de ubicación de los puntos de control de medición.

\section{Toma de datos}

Para realizar las mediciones de velocidad se fijó un punto de referencia desde el cual se inician las mediciones que se muestra en la Figura 4. Al inicio de la medición el vehículo se encuentra detenido, alineando la antena del GPS con el punto de control inicial. Este punto constituyó el km 0 para el inicio del registro de posición con el GPS.

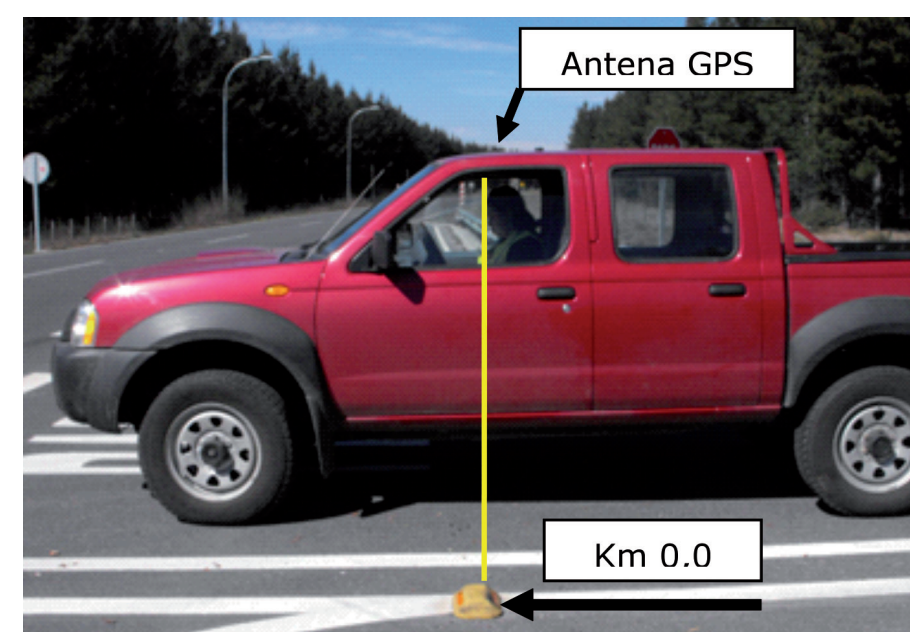

Figura 4: Localización de punto de control inicial y alineación de antena GPS.

El proceso de medición consideró los siguientes pasos:

- Se montó el equipo GPS en el vehículo de prueba. La antena se localizó en el techo, sobre el eje transversal del asiento del conductor y en la mitad del eje longitudinal del vehículo,

- el equipo GPS se programó para que inicie la captura de datos desde el punto en que comienza a moverse,

- los puntos de control se identificaron espacialmente en una primera pasada. Para ello se fijó el odómetro en cero desde el punto de control y se tomaron las distancias medidas desde el $\mathrm{km} 0.0$ hasta cada una de las balizas,

- previo a cada pasada se verificó que no existan vehículos circulando por el tramo de medición, ya que interfieren en la velocidad del vehículo de prueba,

- el conductor inicia su trayectoria acelerando desde 0 $\mathrm{km} / \mathrm{h}$ hasta la velocidad de referencia en la longitud de aceleración y estabilización de velocidad señalado en la Figura 3. Se realizaron pasadas desde los $40 \mathrm{~km} / \mathrm{h}$ hasta los $135 \mathrm{~km} / \mathrm{h}$ cada $20 \mathrm{~km} / \mathrm{h}$,

- el operador de la pistola láser realizó seis mediciones por pasada hasta velocidades de $60 \mathrm{~km} / \mathrm{h}$ y tres mediciones por pasada a velocidad sobre los 60 $\mathrm{km} / \mathrm{h}$. Cada medición se realizó en el momento en que el vehículo de prueba pasaba por las balizas y se corroboraron con las lecturas del equipo GPS. El operador utiliza como punto de medición la placa patente del vehículo. El display de la pistola láser fue filmado durante cada pasada del vehículo de prueba para lograr registrar adecuadamente las lecturas del equipo láser,

- al pasar por cada punto de control, el copiloto registra desde el velocímetro digital del vehículo de prueba la velocidad,

- como se conocen las distancias desde el punto de control inicial hasta las balizas en las que se efectuaron las mediciones, fue posible relacionar la velocidad del vehículo con la posición de cada punto de control y compararla con la velocidad registrada por el copiloto.

\section{Procesamiento de datos}

El procesamiento de datos consideró la depuración de lecturas obtenidas con el GPS mediante el filtro de Kalman, la asociación de valores de velocidad en torno 
a cada baliza y la conformación de la base de datos para realizar las comparaciones. La depuración de las lecturas de velocidad obtenidas con el GPS consiste en eliminar los datos anómalos y acoplar las lecturas de velocidad con la posición registrada en el GPS. El equipo GPS utilizado calcula la velocidad en base al efecto Doppler y para calcular la posición utiliza triangulación con 4 a 8 satélites. Por lo tanto, es necesario acoplar los registros de velocidad y posición. Para ello se utilizó el filtro de Kalman FK implementado en el software del equipo GPS, que es un procedimiento matemático recursivo que permite filtrar señales de datos discretos con el propósito de predecir estados o bien suavizar información con alta variabilidad a partir de una señal medida. El filtro determina el estado de una señal discreta $x_{\mathrm{K}}$ mediante la ecuación (1), a partir de un registro de datos crudo $z_{\mathrm{k}}$ representado por la ecuación (2).

$x_{\mathrm{k}}=A x_{\mathrm{k}-1}+B u_{\mathrm{k}-1}+w_{\mathrm{k}-1}$

$z_{\mathrm{k}}=H x_{\mathrm{k}}+v_{\mathrm{k}}$

En donde $A, B$ y $H$ son parámetros de regresión, $w_{\mathrm{K}-1} \mathrm{y}$ $v_{\mathrm{K}}$ son variables aleatorias que representan el ruido del proceso y de la señal respectivamente. Son mutuamente independientes y siguen una distribución de probabilidades normal con media cero. El FK predice los estados de $z_{\mathrm{K}} y x_{\mathrm{K}}$ minimizando el error cuadrático medio de la covarianza del proceso y eliminando el ruido $w$ y $v$. Los datos de velocidad están representados por la señal $z_{\mathrm{K}}$ y la velocidad resultante se obtiene a partir del valor de $x_{\mathrm{K}}$. Asimismo, con el FK se suavizó la señal de velocidad eliminando datos anómalos originados en la pérdida de calidad de la señal satelital. Posteriormente se acoplaron las mediciones de velocidad y distancia. De este modo se logró contar con registros de velocidad referenciados, a los cuales posteriormente se les asoció la posición de las balizas de cada punto de control.

Para asociar las lecturas de los equipos GPS y pistola láser es necesario seleccionar grupos de datos de velocidad registrados con el GPS en torno a cada punto de control. Puesto que la lectura de la pistola láser es puntual y la del GPS es continua fue necesario tomar 15 datos de velocidad medidos con GPS alrededor de la baliza para obtener un valor de velocidad representativo y comparable con las lecturas de la pistola láser. De este modo, se seleccionaron 15 datos a cada lado de la baliza, totalizando 31 datos para estimar el valor de velocidad media obtenida con GPS.
A estos datos se les asoció en cada baliza el valor de las lecturas de velocidad obtenidas con la pistola láser (5 por punto de control). De este modo se obtuvo una base de datos con valores de velocidad comparables en cada uno de los puntos de control establecidos en el trabajo.

\section{Análisis de resultados}

\section{Comparación de medición de velocidad con tacómetro y equipo GPS}

Esta comparación se realizó con el objetivo de verificar la certeza de la lectura del tacómetro del vehículo de ensayo, de modo que el conductor elija adecuadamente la velocidad de referencia de la medición. Para realizar la comparación, el copiloto registró el valor de velocidad media del GPS y el valor de velocidad registrada con el tacómetro. Para la verificación se utilizó un rango de velocidades entre 20 y $135 \mathrm{~km} / \mathrm{h}$. A estos registros se les aplicó un test de igualdad de media, que arrojó un valor $t=14.5$ y un valor $p=0$, para una significancia del 95\%. Esto indica que las velocidades registradas resultaron estadísticamente diferentes, lo cual justifica la necesidad de homologar las lecturas de velocidad del tacómetro con las del GPS.

La Figura 5 muestra el gráfico con los resultados de la calibración. Se puede apreciar que la relación es absolutamente lineal y que existe un error sistemático de alrededor de $2 \mathrm{~km} / \mathrm{h}$. También se puede visualizar que en la medida que la velocidad de circulación aumenta, la diferencia entre las lecturas de velocidad obtenidas con el tacómetro y con el dispositivo GPS aumentan a una tasa de $1 \mathrm{~km} / \mathrm{h}$ por cada incremento unitario de velocidad. Es decir, a mayor velocidad, mayor diferencia de velocidades entre lectura de tacómetro y de dispositivo GPS.

El resultado obtenido muestra que a $20 \mathrm{~km} / \mathrm{h}$ la diferencia entre el registro del tacómetro del vehículo de prueba y lectura del GPS es de $3.3 \mathrm{~km} / \mathrm{h}$; a una velocidad de 50 $\mathrm{km} / \mathrm{h}$ la diferencia entre ambos aumenta a $5 \mathrm{~km} / \mathrm{h}$ y a 120 $\mathrm{km} / \mathrm{h}$ la diferencia aumenta a $10 \mathrm{~km} / \mathrm{h}$. En todos los casos, la lectura del tacómetro resulta mayor que la registrada con el dispositivo GPS.

Este resultado es relevante para el caso en que se desea calcular el error de medición mediante la técnica de seguimiento vehicular. Si se registra directamente la velocidad del vehículo líder con pistola láser y la del vehículo seguidor con GPS, correlaciones como las 
descritas en la Figura 5 son necesarias para homologar la medición de la pistola láser hacia la lectura del GPS y calcular sobre una misma base de comparación el error de medición.

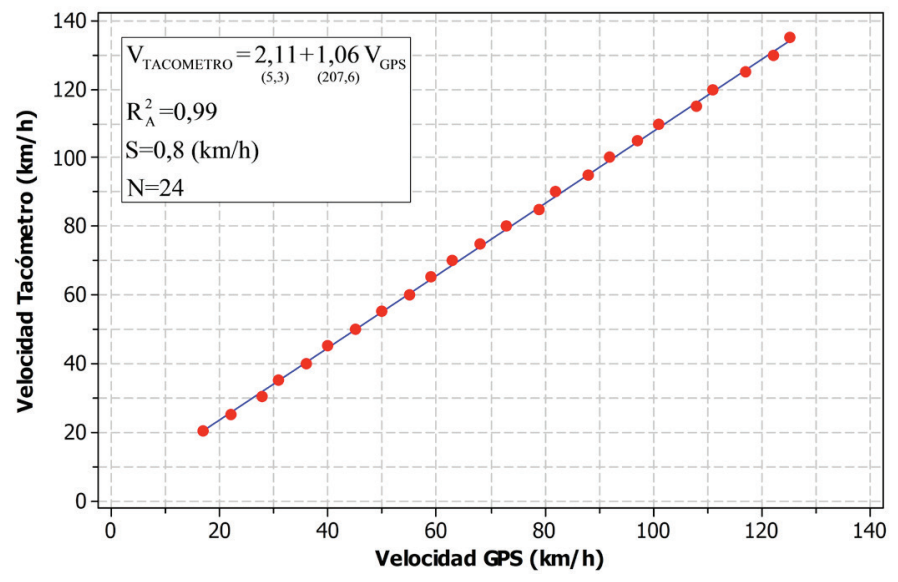

Figura 5: Valores de velocidad medida con tacómetro y con dispositivo GPS

\section{Comparación de velocidad con equipo GPS y pistola láser}

Para realizar esta comparación, se realizaron mediciones de velocidad con velocidades de referencia de 20, 40, $60,80,100$ y $120 \mathrm{~km} / \mathrm{h}$. Puesto que existe una diferencia entre las lecturas de velocidad con GPS y tacómetro, y teniendo en cuenta la calibración de velocidad de ambos, se informó al conductor la velocidad que debía controlar en el tacómetro a partir de los resultados descritos en la Figura 5. Por ejemplo, para una velocidad de referencia de $40 \mathrm{~km} / \mathrm{h}$, el conductor debía conducir a una velocidad del tacómetro de $45 \mathrm{~km} / \mathrm{h}$. Para una velocidad de referencia de $80 \mathrm{~km} / \mathrm{h}$ debía circular hasta una velocidad de $85 \mathrm{~km} / \mathrm{h}$ y así sucesivamente. Posteriormente, con los datos de velocidad procesados se realizó un análisis de varianza (ANOVA) de medidas repetidas para determinar las diferencias de mediciones entre equipos. La Tabla 2 muestra el resumen de los resultados obtenidos con tres grupos de datos vecinos alrededor del punto de control.

De la Tabla 2 se puede concluir que para todos los niveles de velocidad el valor $p$ resultó ser significativo, por lo cual las velocidades medidas con ambos equipos son estadísticamente iguales. La desviación estándar ponderada osciló entre $0.5 \mathrm{~km} / \mathrm{h}$ y $2.9 \mathrm{~km} / \mathrm{h}$, siendo menor a velocidades bajas y mayor a velocidades altas.
Tabla 2: Resumen de ANOVA de registros de velocidad obtenidos con GPS y pistola láser para 5, 10 y 15 datos vecinos.

\begin{tabular}{|c|c|c|c|c|c|c|}
\cline { 2 - 8 } \multicolumn{1}{c|}{} & \multicolumn{6}{c|}{ Número de datos vecinos } \\
\cline { 2 - 8 } \multicolumn{1}{c|}{} & \multicolumn{2}{|c|}{15} & \multicolumn{2}{|c|}{10} & \multicolumn{2}{c|}{5} \\
\hline $\begin{array}{c}\text { Veloc., } \\
\mathrm{km} / \mathrm{h}\end{array}$ & $\begin{array}{c}\text { Valor } \\
p\end{array}$ & $\begin{array}{c}\text { Desviación } \\
\text { estándar } \\
\text { ponderada }\end{array}$ & $\begin{array}{c}\text { Valor } \\
p\end{array}$ & $\begin{array}{c}\text { Desviación } \\
\text { estándar } \\
\text { ponderada }\end{array}$ & $\begin{array}{c}\text { Valor } \\
p\end{array}$ & $\begin{array}{c}\text { Desviación } \\
\text { estándar } \\
\text { ponderada }\end{array}$ \\
\hline 40 & 0.26 & 0.5 & 0.26 & 0.5 & 0.25 & 0.5 \\
\hline 60 & 0.36 & 1.0 & 0.36 & 1.0 & 0.36 & 1.0 \\
\hline 80 & 0.74 & 1.6 & 0.75 & 1.6 & 0.75 & 1.6 \\
\hline 100 & 0.81 & 1.4 & 0.84 & 1.4 & 0.86 & 1.4 \\
\hline 120 & 0.07 & 2.9 & 0.02 & 2.8 & 0.07 & 2.9 \\
\hline
\end{tabular}

La Figura 6 muestra la relación entre las mediciones obtenidas con ambos equipos. Se puede ver que la diferencia de velocidad entre ambos equipos es de carácter sistemático y vale aproximadamente $0.5 \mathrm{~km} / \mathrm{h}$. Esta diferencia se encuentra dentro del intervalo de predicción de la regresión, por lo cual se puede considerar que estadísticamente las mediciones son similares.

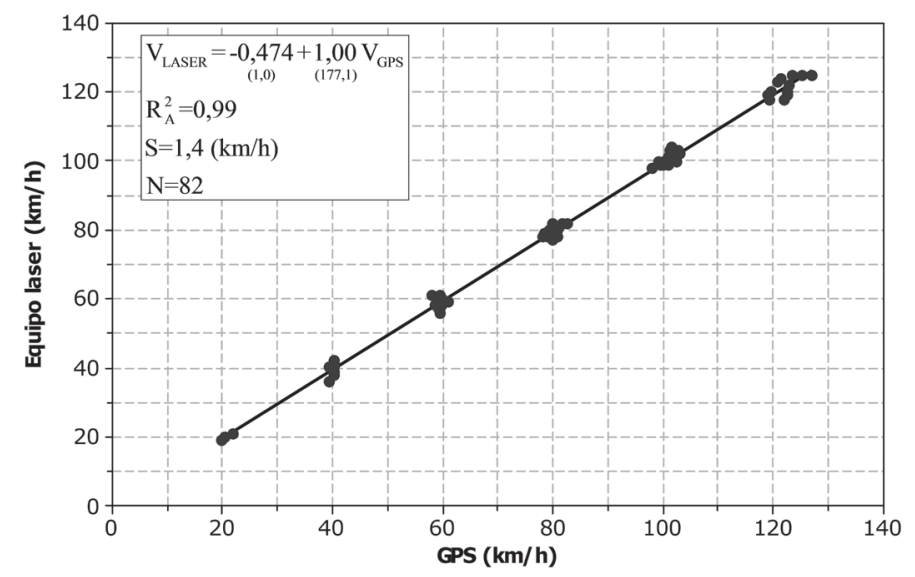

Figura 6: Correlación entre registros de velocidad obtenido con GPS y pistola láser

\section{Conclusiones}

Este trabajo tuvo por objetivo homologar mediciones de velocidad realizadas con tacómetro, dispositivo GPS y pistola láser, con el fin de obtener relaciones entre estos equipos, que permitan a su vez, reducir sesgos en estudios de velocidad. Tanto la pistola láser como el dispositivo GPS se usan para obtener registros de velocidad para modelar su relación con la geometría. Puesto que 
habitualmente se usan en forma combinada, es relevante conocer la relación de lecturas de velocidad entre ambos equipos, a fin de contar con una base de comparación adecuada para mediciones, estudios de precisión y para modelación de relaciones velocidad - geometría. Los resultados obtenidos mostraron que existen diferencias estadísticamente significativas entre las lecturas que arroja el tacómetro del vehículo de prueba y el dispositivo GPS. Diferencias que varían entre $2 \mathrm{~km} / \mathrm{h}$ para velocidades bajas y $10 \mathrm{~km} / \mathrm{h}$ para velocidades del orden de $120 \mathrm{~km} / \mathrm{h}$. Este resultado es relevante para estudios de precisión de equipos de medición de velocidad, puesto que el conductor controla la velocidad de referencia mediante la lectura del tacómetro del vehículo de prueba, la cual no representa la velocidad verdadera de circulación del vehículo, introduciendo un sesgo que no necesariamente es debido al equipo de medición de velocidad en si mismo, sino que debido a la variabilidad de la velocidad de referencia. Asimismo, se determinó que las diferencias entre las lecturas de velocidad realizadas con el dispositivo GPS y la pistola láser son estadísticamente iguales. No obstante, las lecturas realizadas con pistola láser sistemáticamente arrojaron valores $0.5 \mathrm{~km} / \mathrm{h}$ superiores a la registrada con el dispositivo GPS. Este resultado permite homologar mediciones con ambos dispositivos particularmente para el cálculo del error de predicción de velocidad al aplicar el método de seguimiento vehicular.

\section{Agradecimientos}

Los autores de este trabajo desean agradecer al Fondo Nacional de Desarrollo Científico y Tecnológico FONDECYT del Ministerio de Educación de Chile por el financiamiento otorgado al proyecto FONDECYT 11090029, dentro del cual se enmarca este trabajo.

\section{Referencias}

AASHTO (2009). AASHTO guidelines for traffic data programs. American Association of State Highways and Transportation Officials. USA.

Abdelwahab, W.M., Aboul-Ela, M.T. and Morrall, J.F. (1998). Geometric design consistency based on speed change on horizontal curves. Road \& Transport Research 7(1), 13-23
Al-Masaied, H.R., Hamed, M. Aboul-Ela, M. and Ghannam, A.G. (1995). Consistency of horizontal alignment for different vehicle classes. Transportation Research Record 1500, 178-183

Bhuyan, P.K. and Rao, K.V.K. (2012). Defining LOS criteria of urban streets using GPS data: k-means and k-medioid clustering in Indian context. Transport 27(2), 149-157

Cohen, J. (1988). Statistical Power Analysis for the Behavioral Sciences, LA Associates. USA.

Echaveguren, T. y Sáez, J. (2001). Estudio de relaciones velocidad-geometría horizontal en rutas de la VIII región. X Congreso Chileno de Ingeniería de Transporte, 9-12 de Octubre 2001, Santiago, 341-350.

Echaveguren, T. y Basualto, M. (2003). El análisis de aceleraciones en la consistencia de elementos simples de alineamientos horizontales. XI Congreso Chileno de Ingeniería de Transporte, 20-24 de octubre 2003, Santiago, 391-402.

Echaveguren, T. Sepúlveda, P. y Vargas-Tejeda, S. (2011). Evaluación de precisión de mediciones de velocidad de operación en carreteras obtenidas con GPS. XV Congreso Chileno de Ingeniería de Transporte. 3-6 de Octubre 2011. Santiago.

Echaveguren, T. (2012). Análisis de consistencia de caminos bidireccionales usando mediciones continuas de velocidad de operación obtenidas con GPS. Revista Ingeniería de Construcción 27(2), 55 - 70

Echaveguren, T. Arellano, D. y Vargas-Tejeda, S. (2012a). Estudio empírico de velocidad de operación de vehículos pesados en pendientes ascendentes. XVII Congreso Panamericano de Ingeniería de Tránsito, Transporte y Logística. Art \#326, Santiago, Chile

Echaveguren, T., Vargas-Tejeda, S. y García, C. (2012b). Estudio de límites de velocidad en curvas horizontales usando aceleraciones laterales. XVI Congreso Argentino de Vialidad y Tránsito. Art \#20177, 22-26 octubre, Córdoba

Echaveguren, T. and Vargas-Tejeda, S. (2013). A model for estimating advisory speeds for horizontal curves in two-lane rural roads. Canadian Journal of Civil Engineering 40(12), 1234-1243, 10.1139/cjce-2012-0549.

Kustom Signals (2002). Prolaser ${ }^{\circledR}$ III Lidar system specifications. Kustom Signals Inc. USA. 
Li, Y. (2009). Travel time estimation and incident detection using probe vehicles. Use of GPS equipped probe vehicles for traffic data collection. VDM Verlag, Germany

Memon, R., Khaskheli, G.B. and Qureshi, A.S. (2008). Operating speed models for two lane rural roads in Pakistan. Canadian Journal of Civil Engineering 35(5), 443 - 453

Pérez, A.M. García, A. Camacho, F. and D’Attoma, P. (2010). Use of GPS data to model operating speed and deceleration on twolane rural roads. Transportation Research Record 2171, $11-20$
Racelogic (2008). VBOX Mini User Guide. UK.

Schuessler, N. and Axhausen, K.W. (2009). Processing raw data from Global Positioning Systems without additional information. Transportation Research Record 2105, 28-36

Vargas, S. (2008). Definición de umbrales mínimos de resistencia al deslizamiento en pavimentos, en base a un enfoque de demanda y oferta. Tesis Doctoral, Pontificia Universidad Católica de Chile 